\title{
World Health Spatiotemporal Random Variables in Strong Correlation with Mathematical Deterministic Conditions
}

\author{
Persefoni Kritikou $^{1}$, Mihael Maniadhakis ${ }^{2}$ \\ ${ }^{1}$ Health Economist, London, UK \\ ${ }^{2}$ Geographer, Athens, Greece
}

\section{Email address:}

persefoni.kritikou@gmail.com (P. Kritikou), maniadhakis@gmail.com (M. Maniadhakis)

\section{To cite this article:}

Persefoni Kritikou, Mihael Maniadhakis. World Health Spatiotemporal Random Variables in Strong Correlation with Mathematical Deterministic Conditions. Science Journal of Public Health. Special Issue: Spatial Analysis and Mathematics in Health Research, during Times of Global Socio-Economic Instability. Vol. 3, No. 3-1, 2015, pp. 18-23. doi: 10.11648/j.sjph.s.2015030301.14

\begin{abstract}
The present report describes sufficiently documented results of a research undertaken in an effort to detect possible effects of the $21^{\text {st }}$ century's socioeconomic transformation on Health Care Services. Before embarking on such research, presuppositions ought to be examined using the vastly developed methods and techniques in mathematical - statistical research. Results until now lead to a restatement of assumptions, principally on the combination of Set Theoretic and Probabilistic stereotypes.
\end{abstract}

Keywords: Logistic Recurrent Model, GIS, Dependency

\section{Introduction}

The emerging evolution of a socioeconomic transformation, and its potential impact on health care expenditure (considered to be a social service and necessarily a fundamental Human Right) ought to be examined through the view of methods and techniques (stochastic or not) with an attempt to achieve better Health Policy and Social Care. In addition, spatiotemporal locality demands that deterministic explanatory considerations are more extensively used compared to universal random processes.

Previous researchers have set the concept of the randomness of health data in doubt. Generally, it is common that random-like variables are being treated as "chaotic" (mathematically determined by a differential dynamical system), through the detection of long-term spatiotemporal patterns [1].

Having denied the Aristotle's discrimination among the notions of "probable", "possible" and "eventual" as they are declared to be completely independent in "Organon: A Priori Analytics, Book A. 25b", we assume that anything can be "eventual" and "probable" if and only if it is evinced as "possible". Furthermore, if any "eventual" is necessary and defined to be of a deterministic nature as "Ding an sich noumenon" (Immanuel Kant: "Critique of Pure Reason"), then it is of random nature.
The applied linear and stochastic models utilized in Health Care Policies, in case of luck of randomness, are not an appropriate approach when exploring ways to achieve optimization of provided services. Especially in periods of general consumption decline, the practice of restricting the Health Care Expenditure, under the provision of preventing waste of resources and funds may to lead to a mathematical efficiency for a systemic collapse.

The question of whether an existing substructure pattern is a local phenomenon of a random reality or not, may be answered negatively in cases where the reality is by definition deterministic. Therefore logistic models might be investigated and implemented for competitive variables. Specifically, in case of logistic competitive variables, the economic decline is decreasing the whole system capacity; hence, this economic decline is crucial in terms of social profile changes and Health Care Systems' failure.

\section{Materials, Methods and Techniques}

Two types of yearly data, macroeconomic and health data were collected for 193 countries across all continents and for the period 1995-2012.

The macroeconomic variables, collected from the World Bank Databank [2], included data on Gross Domestic Product (GDP) per capita, Consumption, Health Expenditure and 
Total Population on a per country basis.

The health variables, collected from the Global Health Observatory of The World Health Organization [3], included data on major diseases (communicative and noncommunicative) evaluated in years of life lost, for each country individually.

In the case of the mathematical substructure, the probability of health care expenditure as a proportion of total consumption ability, conditional on the years of life lost is of randomlike mathematically determined state.

The geographical data per country were utilised through a GIS system (ArcInfo 9.3). The geodatabase was developed in order to explore whether the geographic spread of human activities under study, was uniform, random or concentrated [4, p230-238].

In addition, the generic macroeconomic variables had to be standardized and weighted by the population structure, the total area, the concentration of human activity and the wealth of each country, using mainly interpolating techniques $[4$, p.320-345].

The variographic [4, p 326-332] methods determined the discrimination ability (i.e. "the nugget") and consequently the white noise or concentrating levels of our data. Besides, the covariance estimation is (by definition) part of the variography process.

The evaluation of health burden in standardized years of life lost was pursued, because it reflected both low quality of life and lack of human resources in the health care sector. The former corresponded to the existential subjective burden, which has an indirect social effect on weakening human activity; the latter corresponded to the direct loss of healthy and socially active people.

\subsection{Analysis of Data}

Our data showed that the annual GDP per capita (World Bank Databank) as well as the general consumption has an increasing trend over the period of 1995-2012, for most of the countries (calculated in United States dollars -USD). Nonetheless, these indicators, after being standardized by the corresponding commodity index and USD index, provided contradictory evidence: a major declining trend is observed from 1995 to 2001, followed by another even steeper declining trend lasting through 2013; presented in Maps 1, 2, 3 and 4 below.

The key outcome however, is not the above-mentioned declining trends in the annual GDP per capita and the general consumption per se. Rather, the investigation of the effect of the private direct household (out-of-pocket) spending for health care services, as a percentage of the consumption ability (given these two macroeconomic variables).

The case of the private direct household (out-of-pocket) spending for health care service, as a percentage of the consumption ability, comprises a competitive variable. As this is in contradiction with the total market access, it is not only a barrier to satisfaction of basic needs, but through feedback, the "predator" of the remaining consumption, up to a point, of course where there will be (if any) a negative effect on it. Consequently, in its nature, the logistic model and the possible implementation of it has to be, de facto, examined. For that reason, the logistic model does not describe any kind of "local substructure efficiency".

As a recurrent process the Logistic Model is simply [5]:

$$
X_{n+1}=r \cdot X_{n} \cdot\left(1-X_{n}\right)
$$

The analysis of these data revealed that $85 \%$ of the countries, comprised a group of cases where the model explained, at the very least, an almost periodic behaviour $(r>2)$; while $54 \%$ of the countries comprised a group of cases where the model explained a randomlike "chaotic" behaviour $(3.75<\mathrm{r}<3.97)$.

\subsection{One Deterministic Variable Driving a Random One}

The fact that non-communicative and communicative diseases are strongly correlated with the GDP per capita and the consumption profile of each country, may give rise to the question of whether the variables are depended or not. In case of independent variables, any correlation is coincidentally explained. And this is true for random stochastic processes.

Hence, because of the descriptive form of deterministic variable's statistics, that has no probabilistic properties, the joint probability can be strongly correlated, irrespective of whether the two variables are dependent or not.

Given the decreasing market access, whatever the feedback to consumption and to the market is, it correlates to the ceiling of the percentage of personal health care expenditure. Additionally, the feedback fastens the approximation to the higher limit of this competitive variable, increasing the complexity of the system.

$$
P(A \backslash B)=\frac{P(A \cap B)}{P(B)}
$$

The notion of mutual information and expectancy in such cases must be considered under the mathematical analysis of uncertainty in such systems and should be defined in a more general way [6], [7].

\section{Results}

The calculated results, presented in the maps below, show a strong positive relation between the group of countries of generally higher consumption ability and prevalence of noncommunicative diseases, such as France, England, Germany, USA and Russia. Instead, the group of countries of generally lower consumption ability, (irrespective of the GDP per capita observed in each country), was related to communicative diseases, such as Central Africa region, and Polynesia (maps 1, 2, 3, 4).

Another effect, in any case, is the correlation of the complex health care expenditure burden (chaotic behaviour) to the group of countries less affected by the GDP per capita decrease in general (maps 5, 6, 7). 
The countries for which the logistic model's almost periodic or randomlike behaviour was examined, seemed to be the "Third World" countries. Even though these countries presume a better adjustment ability to the worldwide economic decline, one could conclude that "the poorer- considered, the better the adjustment".

Almost $54 \%$ of all countries had a logistic parameter $r$ of random like "chaotic" behaviour $(3.75<\mathrm{r}<3.97$ like Niger, Nigeria, Senegal, Zimbabwe, Colombia, Paraguay, Afghanistan, Albania, Azerbaijan, Slovakia, India, China, etc).
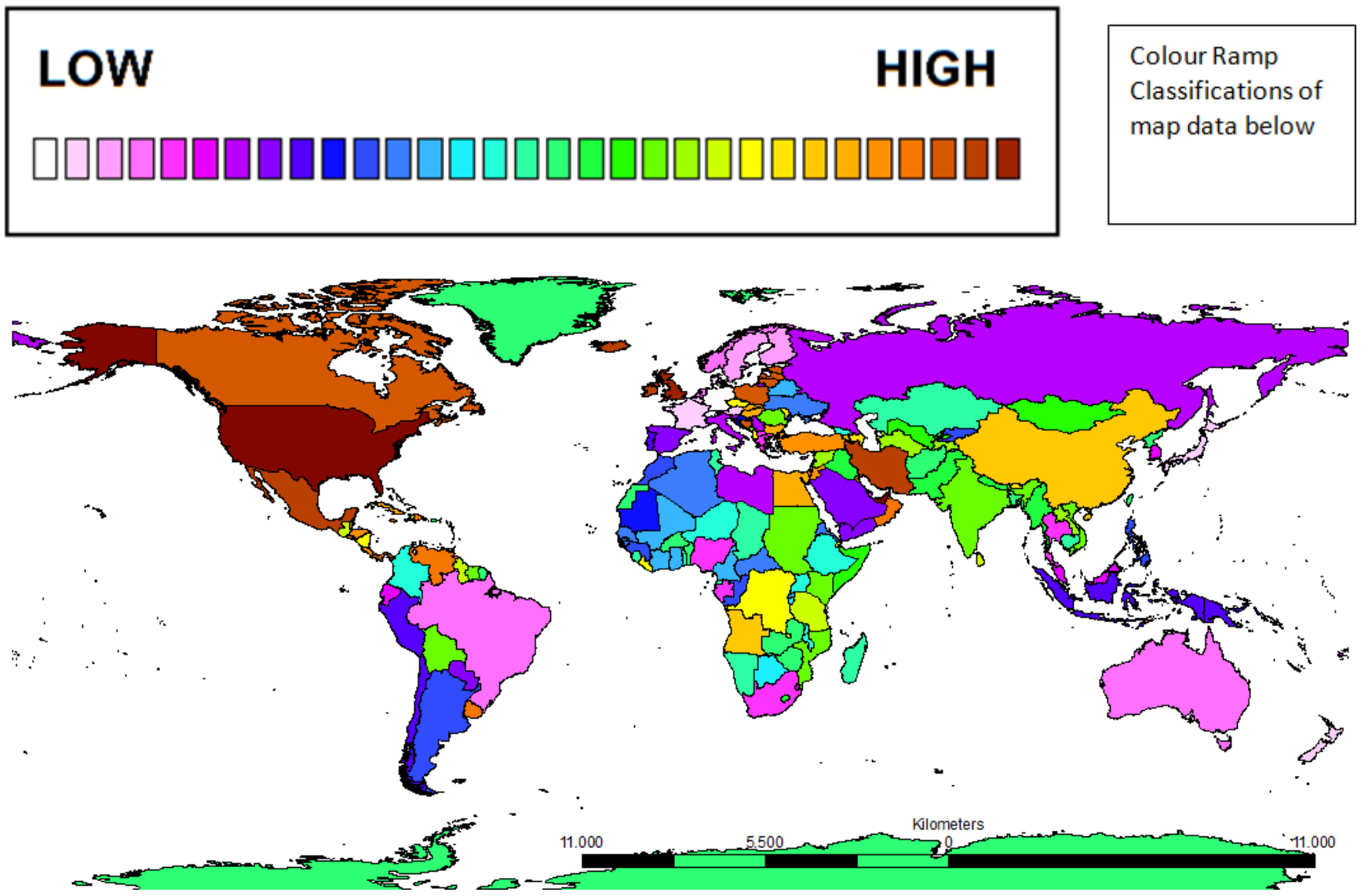

Map 1. Consumption Ability Classification (2001)

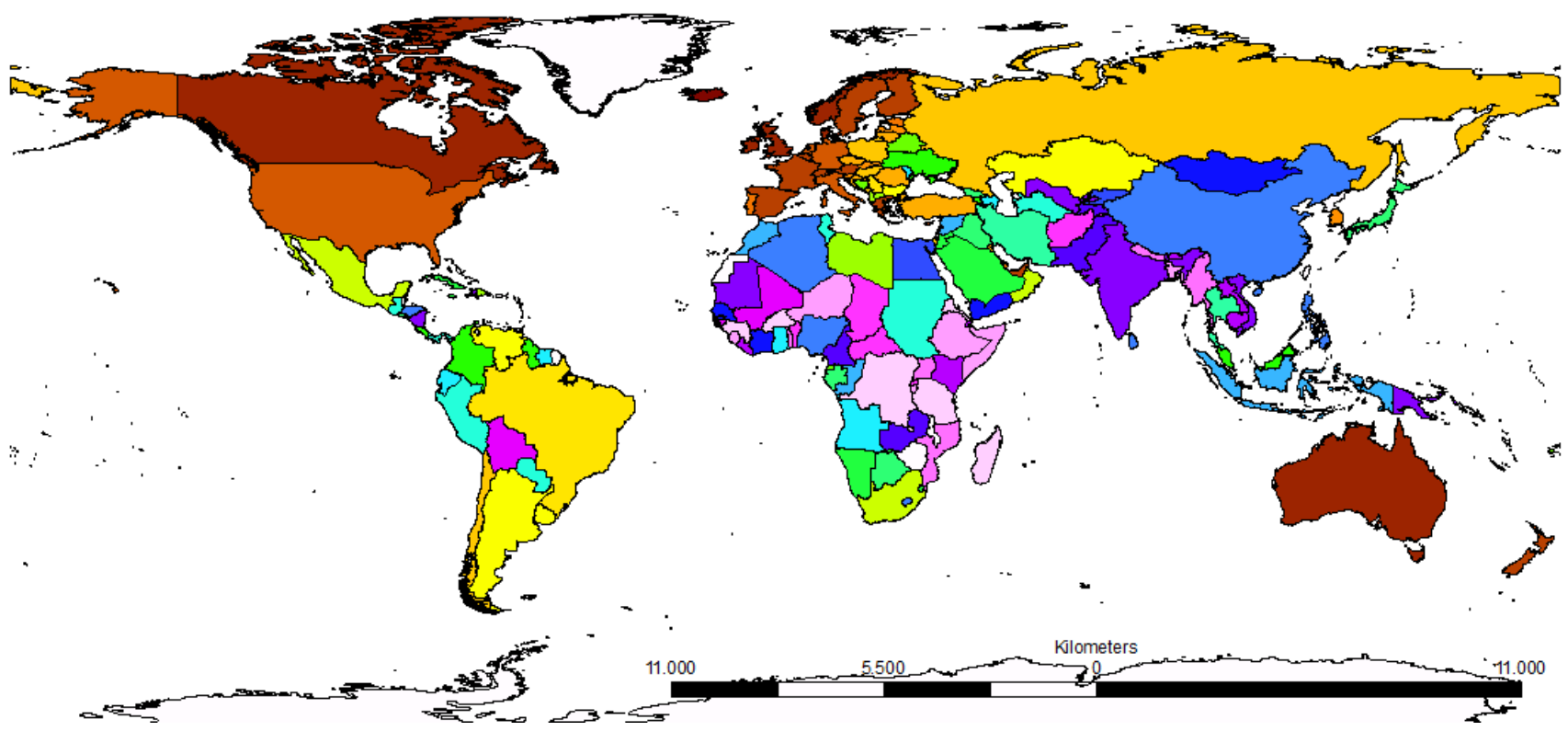

Map 2. Consumption Ability Classification (2012) 

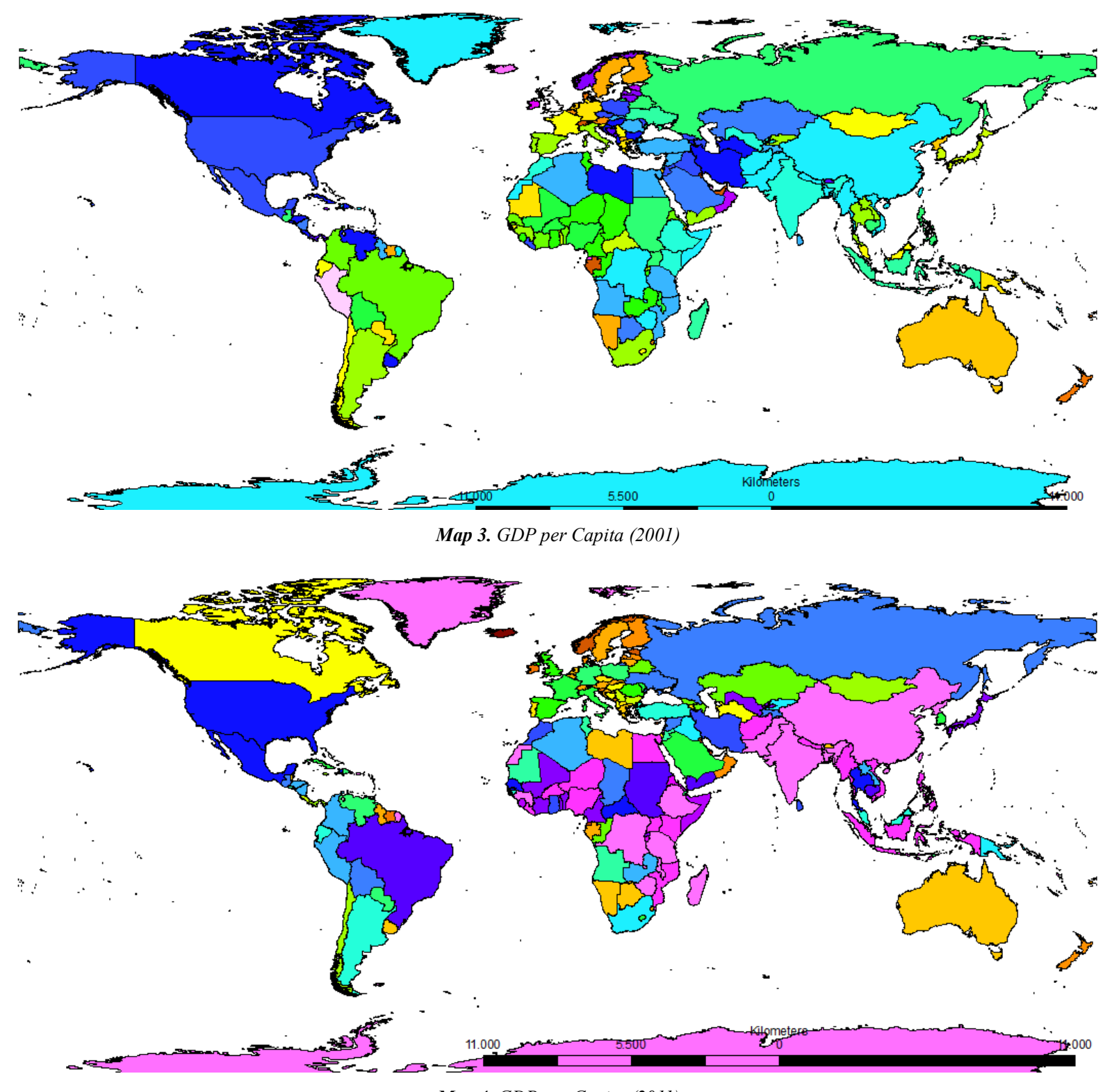

Map 4. GDP per Capita (2011)

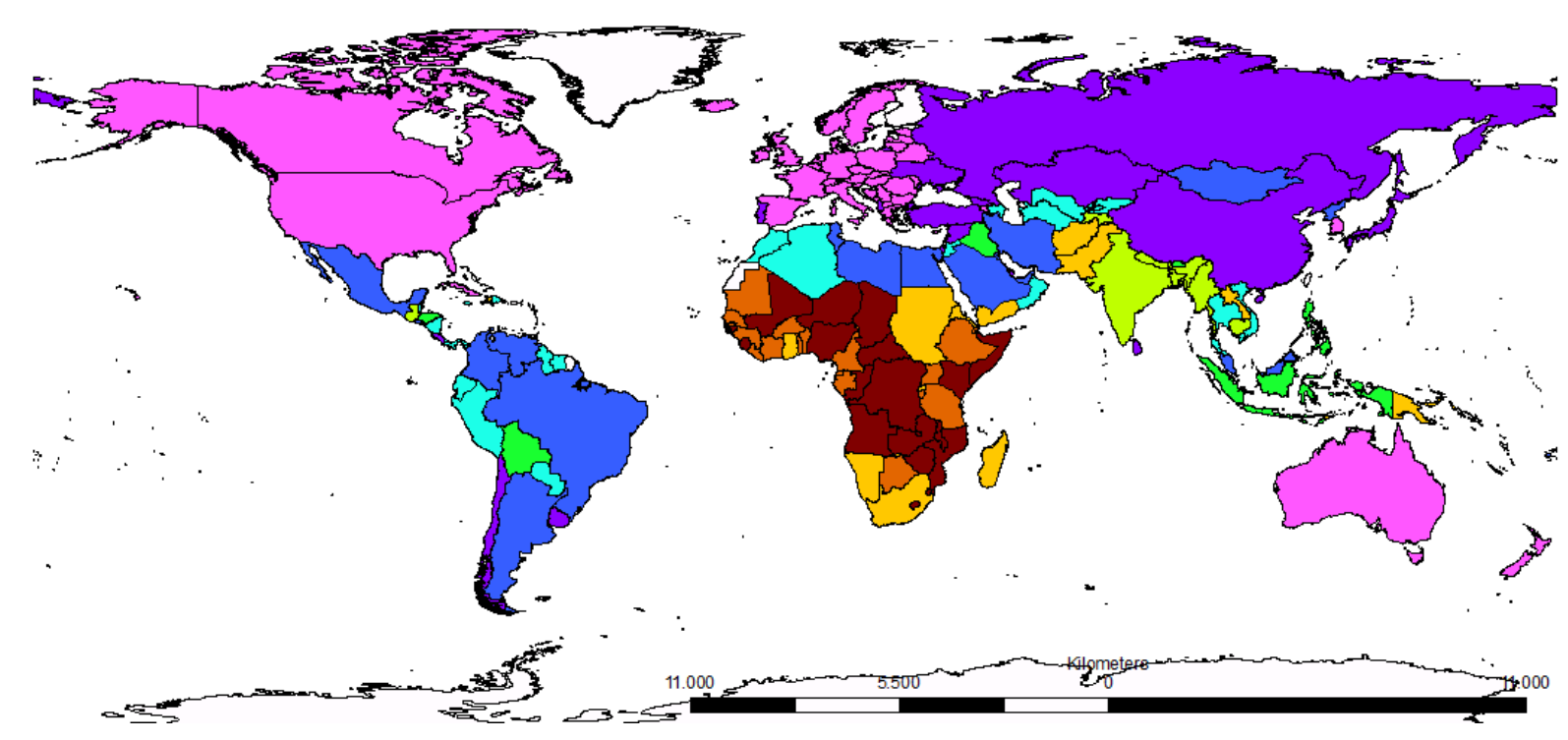




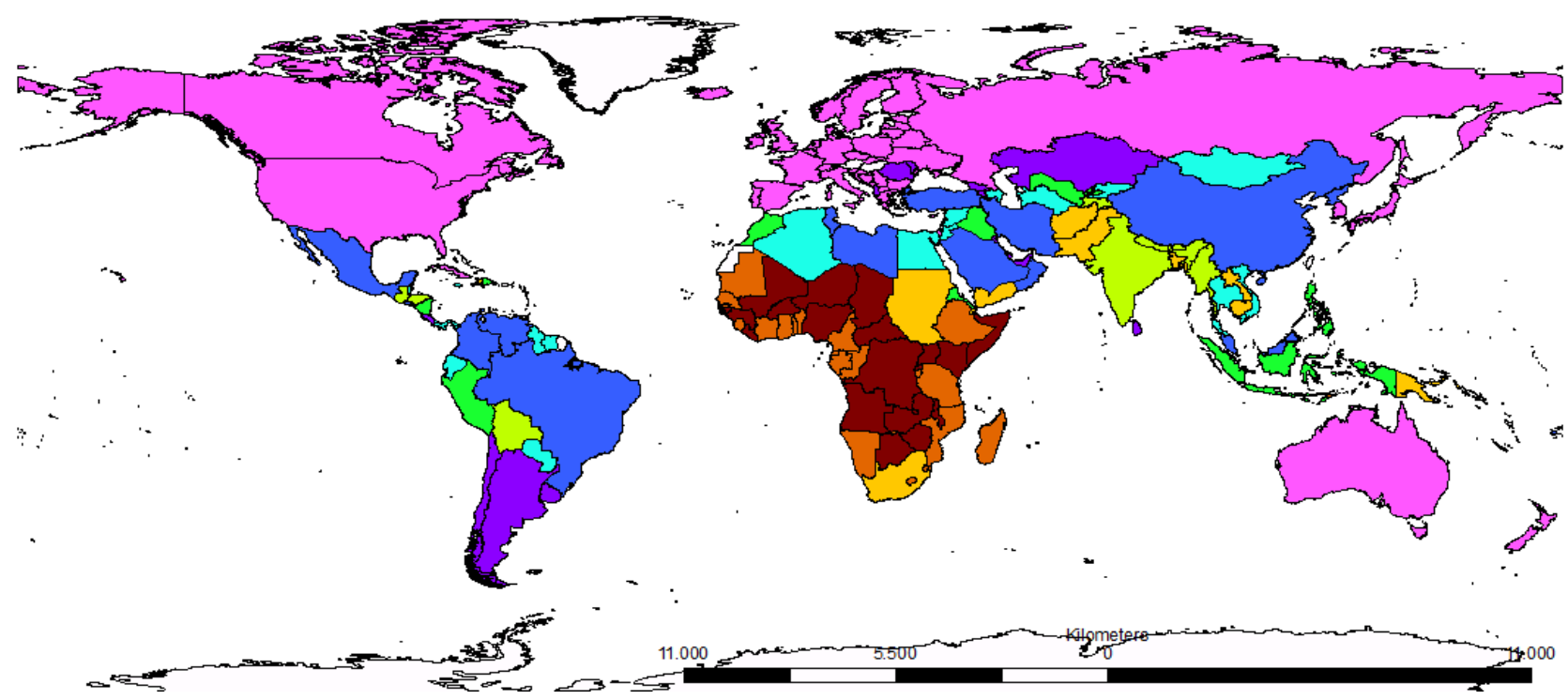

Map 5. Communicative Diseases effect (in lost years) 2002(up), 2012 (down)
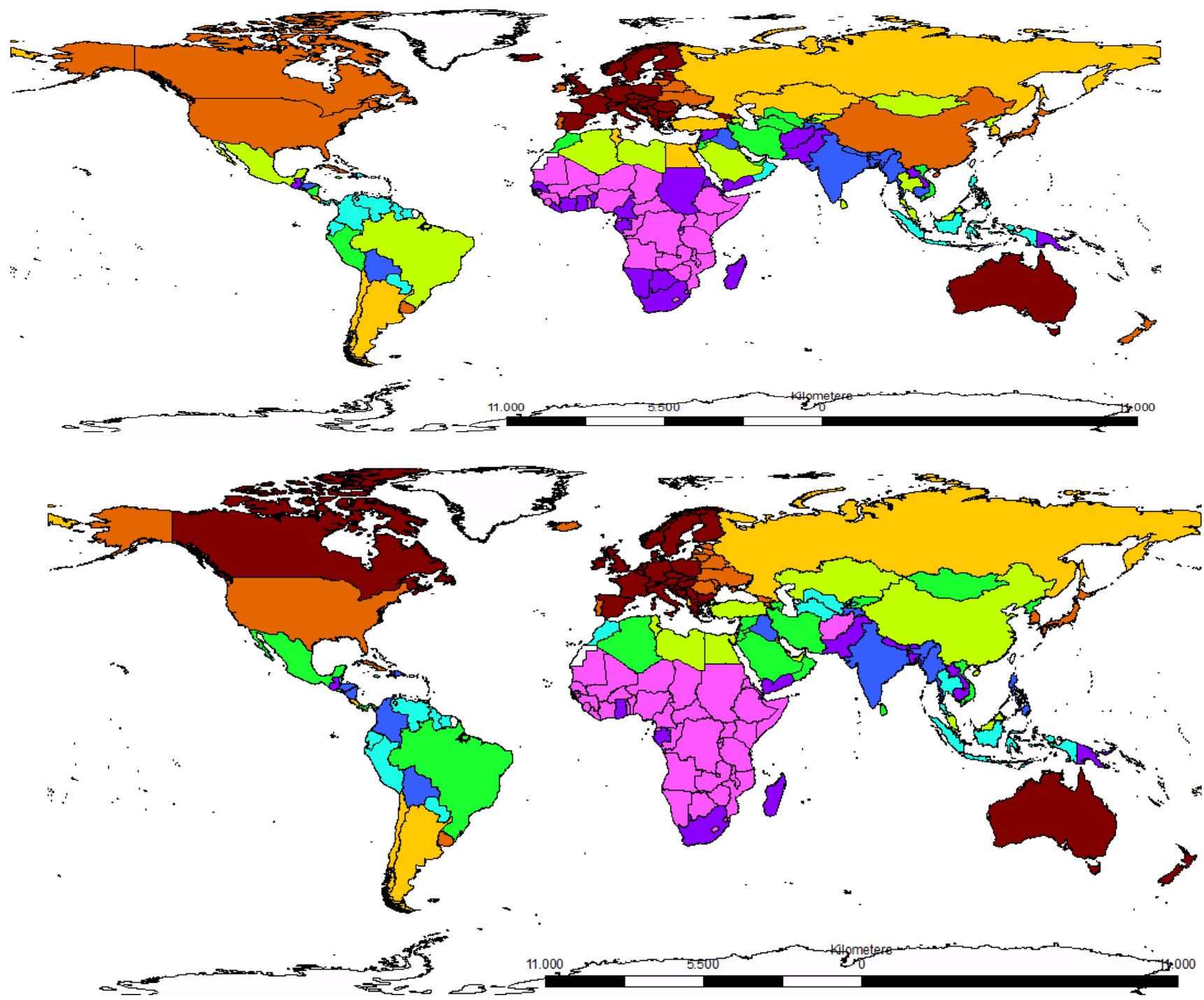

Map 6. Non-communicative Diseases effect (in lost years) 2002(up), 2012 (down) 


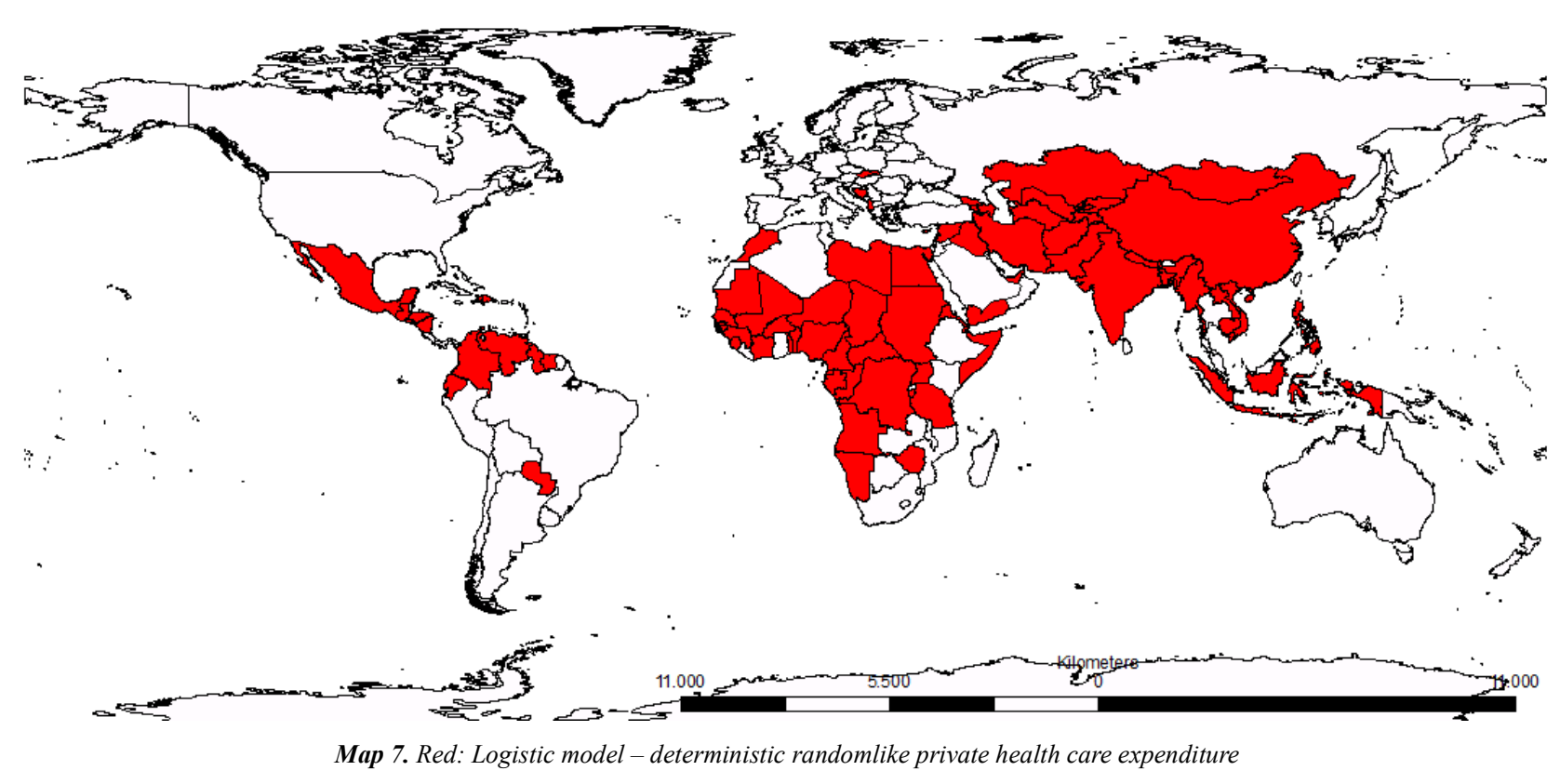

\section{Discussion}

The question under research was the exploration of potentially long-term memory deterministic nature of random like variables. We furthermore investigated the possibility of stable profiling, through instable dynamical systems, and therefore, how this stability may be "maintained" throughout a vast socioeconomic transformation.

Probabilistic nature's variables indicate less sensitivity in small changes and suggest robust linear control, even under the presence of decreasing economic trends.

In case of a deterministic reality, dependency is not a restriction in order to study correlative variables. On the contrary, in case of random variables, correlation can only be studied only if they are dependent. For deterministic variables, correlation is explored under the assumption that a comprehensive realistic framework is present.

The identification of a sufficient logistic model for the Health Policy and Social Care reality implies extreme risk of instability, in case of economic decline, if prevention and health promotion are not funded adequately.

In particular, wherever the central planning restricts economical divergence, and law regulations substitute the major social network reflexes, the aforementioned burden becomes a governmental obligation, due to lack of social sufficiency. It is therefore explained how the "Third World" countries are characterized by a remarkable adjustment ability, despite the fact that their undeveloped Health Care systems cannot accommodate the dangers associated with communicative diseases

\section{Conclusions}

The fundamental outcome of the present study was that further research on the matter is warranted.

The "Universal Locality" Principle [1] is justified and explained as long-term memory in spatial structures implies chaotic behaviour. This behaviour is aggravated when a naturally randomlike deterministic variable is the presupposition of a random-considered one. Hence chaos arises. Dynamical systems are therefore necessary for any further research.

However this spatiotemporal locality demands for the detection of what is the discrimination ability level. Any human, as an instance in both Space and Time, implements a function of possible situations of a transfinite number of scores. Through the combinatorics of interrelated variables of transfinite number of values $(0 \ldots 1)$, the Cantorian Set Theoretic ordinal algebraic methods [8] seem to be a challenging but promising necessity.

\section{References}

[1] Pistolla G,Prastakos P, Vassilaki M, Philalithis A, 2010. Spatial-Mathematic methods for analysis of indicators of mortality. IJAEST- Vol No. 1, Issue No. 2, 135 - 146.

[2] World Bank Databank, http://data.worldbank.org/

[3] http://apps.who.int/gho/data/?theme=main

[4] Koutsopoulos Kostis, "Geographic information systems and spatial Analysis” Papasotiriou, Athens, 2002

[5] Bountis Anastasios, "Dynamic systems and Chaos", Papasotiriou, Athens, 1995

[6] Kolmogorov, Andrey (1963). "On Tables of Random Numbers". Sankhyā Ser. A. 25: 369-375

[7] Kolmogorov, Andrey (1998). "On Tables of Random Numbers". Theoretical Computer Science 207 (2): 387-395

[8] Cantor, Georg "Contributions to the Founding of the Theory of Transfinite Numbers", Trochalia, Athens, 1998 